\title{
Use of traditional Chinese medicine in HIV/AIDS in China*
}

\author{
Jian Wang, Wen Zou, Ying Liu
}

Traditional Chinese Medicine Center for AIDS Prevention and Treatment, China Academy of Chinese Medicine Sciences, Beijing, China.

Email: 62tiger@163.com

Received 20 May 2010; revised 8 June 2010; accepted 22 June 2010.

\begin{abstract}
This paper gives a general introduction of HIV/AIDS treatment with Traditional Chinese Medicine (TCM) in China during the past 20 years. Although the role of TCM in treatment of HIV/AIDS is promising, there is still a long way to go.
\end{abstract}

Keywords: HIV/AIDS; Traditional Chinese Medicine (TCM)

\section{INTRODUCTION}

Since the early 1980's over 40 million individuals have infected with HIV worldwide and over 12 million have died. In China there had by 2009 been 319,877 cases were identified as HIV/AIDS [1]. The medical, social and economic implications of HIV infection are devastating.

In 1996, protease inhibitor therapy became widely available for use in the treatment of HIV infection, thus a decline in HIV-related morbidity and mortality had been reported. Over the last decade, more and more Chinese HIV/AIDS patients turn to seek TCM treatment, among the reasons commonly cited for the use of TCM by patients with HIV infection are: expectation of good effect, reduction of symptoms from the disease or reduction of medicine side effect, or desire for improved quality of life and increased control over the disease process. In all, there is a growing need in china for TCM to support people living with HIV/AIDS, to help to alleviate some of the side effects of the drug therapy, to improve quality of life and to assist in the treatment of individuals who are either unwilling, unresponsive or resistant to combination therapy.

This paper gives a general introduction of use of traditional Chinese medicine in HIV/AIDS in China over the past 20 years.

*Granted Fund: State Major Science \& Technology Specific Projects (2008ZX10005-001)

\section{TCM VS AIDS}

TCM is a complete medical system with its own unique philosophy, diagnostics and treatment methods which is different from conventional therapy. As a kind of medical system with thousands years of history, the reason why TCM could exist after such a long time is efficacy in clinical practice that help ancient people alive under arduous climate conditions. The basis of TCM theory is Yin-yang and five elements which related to the whole universe, so TCM views the human body and nature as integral not divided. A healthy human is under the state of relatively dynamic balance maintained by both inside and outside of the body, if the balance is broken, the illness occurs. TCM aims to recover the balance of human body, just as the old saying in TCM bible "The Yellow Emperor's Classic of Internal Medicine”: once Yin balances with Yang, there comes the harmony of the body and the spirit.

Syndrome differentiation and integral regulation are two main characteristics of TCM. TCM focuses on differentiation of syndromes with the holistic and dynamic perspective and aims to the patient with suffering disease instead of only the disease itself. The constitution of the patient is different, the illness phase is various, the body response is diverse, the manifestation is not the same, and therefore the treating principle should be varied.

Because AIDS is a "new" disease, having only been identified in 1981, there is no specific research on the disease from the past or in the classics. TCM does possess a large body of information about infectious diseases and epidemics, and ways to describe the symptoms of those suffering from AIDS. AIDS can be defined in several different ways according to TCM pattern identification. TCM uses the term "yi bing" (infectious epidemic diseases), to describe epidemic diseases such as AIDS, which are caused by invading evils (viruses). AIDS can also be thought of as a "pestilence," The Chinese term for this is "wen yi", such a disease was thought to have a long latency, sudden onset, and severe symptoms. TCM methods used for treating AIDS is di- 
verse, such as herbal medicine, acupuncture and moxibustion, Qigong, which aims to enhance immune function of the body, delay the development of disease, reduce or remove the symptom-signs, so as to improve quality of life and prolong life expectancy. The purpose of TCM is to support the strong energy and body resistance so as to inhibit the progress of disease. Someone has enough strong energy and body resistance in TCM, even if infected with HIV, he can live with it, being a long term HIV carrier, so as to postpone onset and death. In all, from our point of view, the best time of intervening in AIDS with TCM is: For asymptomatic stage (HIV carrier) patients, the key aim of TCM is to maintain and enhance the immune-function so as to delay its progression to AIDS stage. For AIDS stage, TCM treatment focuses on relieving symptoms of patients who have AIDS-related opportunistic infection so as to improve the quality of life or combines with HAART therapy to alleviate the side effect of biomedical drugs.

\section{WHERE IS THE EFFECTIVE EVIDENCE?}

\subsection{Chinese Herbal Medicine Screening}

Tens of thousands of herbs have been screened for anti-HIV activity. More than 140 kinds of herbs demonstrated to harbor HIV inhibitory activity, such as Radix Arnebiae, Herba Violae, Flos Lonicerae, Flos Chrysanthemi Indici, Fructus Mume, Cortex Cinnamomi, Fructus Ligustri Lucidi et al. [2-5] Some bioactive materials extracted or isolated from medicinal herbs showed inhibition of HIV activity as well: polysaccharides (such as polysaccharides of Radix Ginseng, Glossogyne tenuifolia Cass, Radix Astragali, Ganoderma Lucidumseu Japonicum, Radix Actinidiae); protein components (such as glucoprotien in Aloe, a component from Radix Trichosanthis, Alph and Beta Momordicines in Momordica charantia, Ricin bond A, cimigenol saponin); alkaloids (such as castanospermine and colchicines); lactones(such as baicalein and hypericin); terpenes(such as glycyrrhizin and goddypol) et al. Mechanisms of action included inhibition of reverse transcriptase, protease inhibition and integrase inhibition or interference of infection at the viral cell entry level. Among them, Trichosanthin is a kind of prospective one that acquired worldwide attention, it was shown to inhibit HIV replication in infected cells of lymphocyte and mononuclear phagocytic lineage, with no measurable toxicity in uninfected cells, and it was applied in the treatment of patients with AIDS or AIDS-related complex in phase I and II studies [6-11].

Many more herbs or herb extracts may attenuate the course of HIV infection via immune enhancement, cytokine or other pathways, such as Radix Astragali, Cordy- ceps been reported to enhance helper lymphocyte $\mathrm{T}$ and promote CD4/CD8 ratio. [12-13] Shikonin was shown to decline the expression of CCR5 and CCR5mRNA [14].

Many herbal compounds have been tested to have potent HIV inhibitory activity and their experimental or clinical manifestation may provide valuable leads for further investigations. For instance, Xiao chaihu tang, [15] Zhongyan-1, [16] Zhongyan-2, Zhongyan-4, Compound SH [17], Tangcao Tablets et al.

\subsection{Clinical Trials}

According to the regulation of State Food and Drug Administration (SFDA) in China, new Chinese patent medicine treating AIDS can be classified into anti-HIV patent that testified through in vitro and in vivo experiments and patent supplementary for AIDS treatment that either adds synergistic effect or alleviates toxic-side effect on antiretroviral drugs already existed. Tangcao Tablets is the first patent approved by SFDA for alleviating symptoms and signs of HIV/AIDS patients, 5 other relatively matured compounds (Qiankunning Tablets, Keaite capsule, Chuankezhi injection, Zhongyan-2 Granule and Compound Sanhuangsan) are still under clinical trials.

Tangcao Tablets: Wu Hao et al. [18] recruited 173 HIV/AIDS participants for randomized double blind placebo controlled clinical study in 5 medical centers for 6 months, result showed that Tangcao Tablet could significantly raise CD4 count, increase weight and CD4/ CD8, and it was possible to delay the replication of HIV.

Zhongyan-4 Granule: Wang Jian et al. [19] recruited 72 HIV/AIDS participants for a randomized double-blind placebo-controlled trial for 6 months. Results showed that 15 out of 30 patients in Zhongyan-4 group had their CD4 counts increased by $7.70 \pm 150.96 / \mathrm{mm}^{3}$, markedly higher than that in placebo group, no adverse reaction was found.

Qiankunning Tablets: Shi Dan et al. [20] recruited 36 HIV/AIDS participants for a randomized double-blind placebo-controlled trial for 7 months. The results showed that significant difference of plasma HIV-1RNA level was found between patients treated with Qiankunning Tablet and those treated with placebo for control after they were medicated for 7 months, suggesting the Qiankunning tablet was effective in reducing viral load. Moreover, in the next 3 months open treatment, it illustrated further the evident effect of Qiankunning Tablets in reducing plasma level of HIV-1RNA, and raising CD4 dose-dependently. Symptoms and QOL in patients were improved as well.

Xiaomi Granule: Chen Jizhong [21] recruited 34 HIV/AIDS patients with oral candidiasis for a two weeks RCT trial, Participants were randomized into two groups, the 34 cases in treatment group received Xiaomi Granule, 
and the 34 cases in control group received Nystatin. The result showed that Xiaomi Granule group had better effect than the controlled group, and a lower reoccurrence rate was found after two weeks follow-up.

\subsection{Medical Care}

TCM intervention on HIV/AIDS treatment started from 2004, a pilot project named National Free TCM HIV/ AIDS Treatment Program had been launched by The State Administrative Bureau of Traditional Chinese Medicine, and quickly scaled up from 5 provinces (Henan, Hebei, Anhui, Hubei, Guangdong) to 19 provinces, autonomous regions, and municipalities in China including some places with high prevalence, 9267 cases have been treated with TCM accumulatively. The therapeutic effects on 8946 cases from pilot project are as follows: most of the cases maintained stable immune function; main symptoms and signs like fever, cough, fatigue, poor appetite, diarrhea had significantly been improved $(P<0.001)$, no adverse reaction was found in TCM treatment.

\section{HOW TO EVALUATE THE EFFECTIVENESS?}

Current used effectiveness evaluation gold standard: viral load and CD4+T cell count seemed to be not proper for TCM. Some AIDS patients had improved quality of life or prolonged life after TCM treatment, but their viral load might be still in a high level and CD4+T cell count can't see significant elevation. As to patients, improvement in quality of life rather than biomedical parameters changes matters so much to them. So if TCM clinical effectiveness evaluation take items such as patients reported outcomes (PRO), quality of life, clinical end points (morbidity, mortality, life span), activities in daily life (work ability) et al. into consideration, or even let those items play key part in the evaluation, that might be more appropriate for TCM evaluation.

From 2008, China State Major Science \& Technology Specific Projects granted about 100 million on TCM research on HIV/AIDS, which posed big opportunity to TCM researchers. In other words it is also big challenge. Although TCM appears to be associated with improvements in immune function, quality of life, and some AIDS related opportunistic diseases. Definitive conclusions were limited due to variation in designs, comparisons, heterogeneous outcomes and inadequate controls. High-quality, well-controlled, longer randomized trials are needed to better inform clinical decisions in the coming works.

\section{REFERENCES}

[1] Wang, L., Wang, N., Wang, L., Li, D., Jia, M., Gao, X., et al. (2009) The 2007 estimates for people at risk for and living with HIV in China: Progress and challenges. Journal of Acquired Immune Deficiency Syndromes, 50(4), 414-418.

[2] Luo, S.D. and Ju, P. (2006) Compared traditional Chinese medicine and western medicine to treat AIDS. Journal of Henan University of Chinese Medicine, 21(3), 1-3.

[3] Lam, T.L., Lam, M.L., Au, T.K., et al. (2000) A comparison of human immunodeficiency virus type-I protease inhibition activities by the aqueous and methanol extracts of Chinese medicine herbs. Life Science, 67(23), 2889-2896.

[4] Au, T.K., Lam, T.L., Ng, T.B., et al. (2001) A comparison of HIV-1 integrase inhibition by aqueous and methanol extracts of Chinese medicine herbs. Life Science, 68(14), 1687-1694.

[5] Kobayashi, Y., Watanabe, M., Ogihara, J., et al. (2000) Inhibition of HIV-1 reverse transcriptase by methanol extracts of commercial herbs and spices. Journal of the Japanese Society for Food Science and Technology, 47(8), 642-645.

[6] McGrath, M.S., Santulli, S. and Gaston, I. (1990) Effects of GLQ223 on HIV replication in human monocyte/ macrophages chronically infected in vitro with HIV. AIDS Research and Human Retroviruses, 6(8), 1039-1043.

[7] McGrath, M.S., Hwang, K.M., Caldwell, S.E., et al. (1989) GLQ223: An inhibitor of human immunodeficiency virus replication in acutely and chronically infected cells of lymphocyte and mononuclear phagocyte lineage. Proceedings of the National Academy of Sciences, 86(8), 2844-2848.

[8] Byers, V.S., Levin, A.S., Malvino, A., et al. (1994) A phase II study of effect of addition of trichosanthin to zidovudine in patients with HIV disease and failing antiretroviral agents. AIDS Research and Human Retroviruses, 10(4), 413-420.

[9] Mayer, R.A., Sergios, P.A., Coonan, K., et al. (1992) Trichosanthin treatment of HIV-induced immune dysregulation. European Journal of Clinical Investigation, 22(2), 113-122.

[10] Byers, V.S., Levin, A.S., Waites, L.A., et al. (1990) A phase I/II study of trichosanthin treatment of HIV disease. AIDS, 4(12), 1189-1196.

[11] Kahn, J.O., Kaplan, L.D., Gambertoglio, J.G., et al. (1990) The safety and pharmacokinetics of GLQ223 in subjects with AIDS and AIDS-related complex: A phase I study. AIDS, 4(12), 1197-1204.

[12] Gai, L., Song, C.Q., Hu, Z.B., et al. (2001) Review of anti-HIV plants. Foreign Medical Sciences, 23, 139.

[13] He, J.H. and Mai, E.D. (2004) Review of AIDS treatment with Chinese medicine. Chinese Pharmaceutical and Clinical Research, 4, 454.

[14] Chen, X., Yang, L., Zhang, N., et al. (2003) Shikonin, a component of Chinese herbal medicine, inhibits chemokine receptor function and suppresses human immunodeficiency virus type 1. Antimicrobial Agents and Chemotherapy, 47(9), 2810-2816.

[15] Zhang, Z.J. (1995) The phagocytosis of HIV infected cells by Xiao chaihu tang extracts. Foreign Medical Sciences, 17, 64.

[16] Guan, C.F. (1995) Experimental study of SIV infected monkey model treatment by Zhongyan-1. Chinese Jour- 
nal of Information on Traditional Chinese Medicine, 2, 42-46.

[17] Kusum, M., Klinbuayaem, V., Bunjob, M., et al. (2004) Preliminary efficacy and safety of oral suspension $\mathrm{SH}$, combination of five Chinese medicinal herbs, in people living with HIV/AIDS; the phase I/II study. Journal of the Medical Association of Thailand, 87(9), 1065-1070.

[18] Wu, H., Zhang, F.J., Yao, C., et al. (2004) Clinical study of Tangcao tablet in treating HIV/AIDS. Compilation of Theses of Treatment and Research on AIDS, 3, 95-103.

[19] Wang, J., Yang, F.Z., Zhao, M., et al. (2006) Randomized double-blinded and controlled clinical trial on treatment of HIV/AIDS by Zhongyan-4. Chinese Journal of Integrative Medicine, 12(1), 6-11.

[20] Shi, D. and Peng, Z.L. (2003) Randomized double blind placebo paralleled clinical research on HIV/AIDS with Qiankunning tablets. Study Journal of Traditional Chinese Medicine, 21, 1472-1474.

[21] Chen, J.Z. (2009) Xiaomi granule on HIV/AIDS patients with oral candidacies. Guangming Journal of Chinese Medicine, 24, 633-636. 